\title{
CrystEngComm
}

\section{Phase transition between cubic and monoclinic polymorphs of the tetracyanoethylene crystal: the role of temperature and kinetics $\dagger$}

\author{
Bohdan Schatschneider, ${ }^{* a}$ Jian-Jie Liang, ${ }^{b}$ Sebastian Jezowski ${ }^{c}$ and Alexandre Tkatchenko ${ }^{d e}$ \\ Received 6th March 2012, Accepted 20th April 2012 \\ DOI: $10.1039 / \mathrm{c} 2 \mathrm{ce} 25321 \mathrm{f}$
}

\begin{abstract}
Prediction of the relative stabilities and phase transition behavior of molecular crystalline polymorphs is highly coveted as distinct phases can possess different physical and chemical properties while having similar energies. Crystalline tetracyanoethylene (TCNE, $\mathrm{C}_{6} \mathrm{~N}_{4}$ ) is known to exhibit rich solid state phase behavior under different thermodynamic conditions, as demonstrated by a wealth of experimental studies on this system. Despite this fact, the role of temperature and kinetics on the phase diagram of TCNE remains poorly understood. Here, first-principles calculations and highresolution Fourier-transformed infrared (HR-FTIR) spectroscopy experiments are used to study the relative stabilities of the cubic and monoclinic phases of TCNE as a function of temperature.

Specifically, density-functional theory with the van der Waals interactions method of Tkatchenko and Scheffler $(\mathrm{DFT}+\mathrm{vdW})$ is employed. The accuracy of this approach is demonstrated by the excellent agreement between the calculated and experimental structures. We find that the cubic phase is the most stable polymorph at $0 \mathrm{~K}$, but becomes less favorable than the monoclinic phase at $160 \mathrm{~K}$. This temperature-induced phase transition is explained on the basis of varying close contacts and vibrational entropies as a function of temperature. These findings are supported by a temperaturedependent HR-FTIR linewidth study of the $\mathrm{C} \equiv \mathrm{N}$ vibrons.
\end{abstract}

\section{Introduction}

Molecular crystals usually have several polymorphic forms which often possess different physical and chemical properties, but are typically very close in energy. The small energetic gaps between different structural motifs make the prediction of polymorphic transitions very challenging, and sets the precedent for continuous improvement of atomistic modeling methods which can eventually be used for crystal engineering. ${ }^{1-4}$

Crystalline tetracyanoethylene (TCNE, $\mathrm{C}_{6} \mathrm{~N}_{4}$ ) possesses rich solid state behavior, exhibiting many polymorphs. Being the strongest $\pi$-acid, ${ }^{5}$ it also acts as a unique electron acceptor in many key charge-transfer complexes. As a result of TCNE's singular properties, it has been extensively investigated from both basic and applications stand points, e.g. it has been used as a fundamental system for solid state phase transition ${ }^{6-8}$ and

${ }^{a}$ Department of Chemistry, The Pennsylvania State University, FayetteThe Eberly Campus, 1 University Dr, Uniontown, PA, 15401, USA. E-mail: bxs54@psu.edu

${ }^{b}$ Accelrys, Inc., 10188 Telesis Court, Suite 100, San Diego, CA, 92121, USA

${ }^{c}$ Department of Chemistry, The University of California, Riverside, CA, 92501, USA

${ }^{d}$ Fritz-Haber-Institut der Max-Planck-Gesellschaft, Faradayweg 4-6,14195, Berlin, Germany

${ }^{e}$ Department of Chemistry, Pohang University of Science and Technology, Pohang 790-784, Korea

$\uparrow$ Electronic supplementary information (ESI) available. See DOI: $10.1039 / \mathrm{c} 2 \mathrm{ce} 25321 \mathrm{f}$ ionization energy investigations. ${ }^{9}$ TCNE has also served as a vital component in both organic superconductors (Bechgaard salts) ${ }^{10}$ and molecule-based magnets. ${ }^{11}$ Here we concentrate on understanding the fundamental properties that dictate the phase behavior of crystalline TCNE, with an outlook to study the more complex materials mentioned above.

To date, four polymorphic forms of TCNE have been reported, ${ }^{12-22}$ where only the monoclinic and cubic polymorphs have ever been resolved. These different crystal symmetries are accessible via variations in the temperature, pressure, and crystallization conditions. The monoclinic and cubic structures, accompanied by their Hirshfeld surfaces (discussed in detail in Section 3.3), are presented in Fig. 1. Growth of the cubic phase has only been reported from slow evaporation of ethyl acetate below room temperature. ${ }^{12-15}$ In contrast, the monoclinic phase can be grown in a variety of ways: it can be grown from a) ethyl acetate at room temperature ${ }^{16,17}$ b) sublimation (vapor deposition) ${ }^{18,19}$ c) ethyl acetate/carbon tetrachloride solution at room temperature ${ }^{14,20}$ or d) via rapid crystallization in ethyl acetate at low temperatures suitable for cubic growth. ${ }^{17}$ A third phase of hexagonal/trigonal symmetry was reportedly grown from a methylene chloride/ethyl acetate solution at room temperature. ${ }^{20}$ As evident from the wide variety of phases grown by slight variations in the crystallization conditions, the thermodynamics and kinetics involved in TCNE solidification is complex.

Once crystallized, TCNE exhibits pressure- and temperatureinduced polymorphic phase transitions. The temperature-induced phase transition is first order, appearing as an irreversible (down 
Cubic

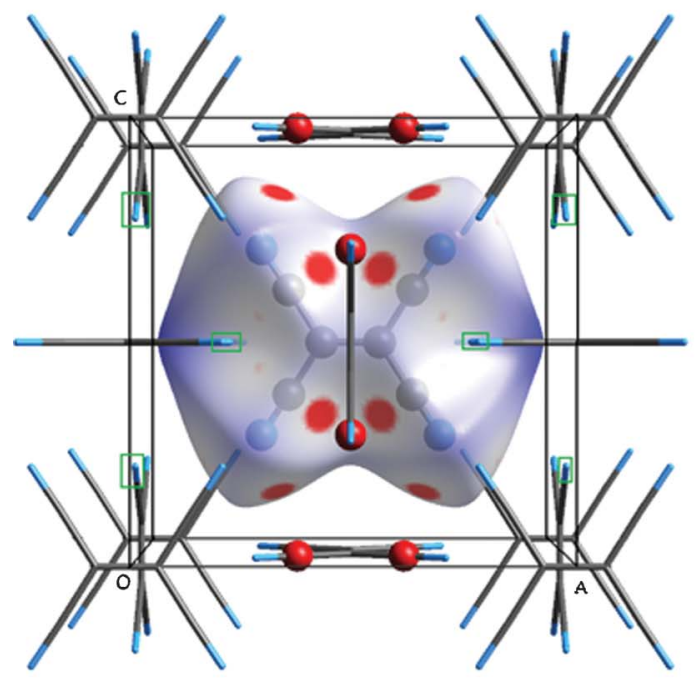

Monoclinic

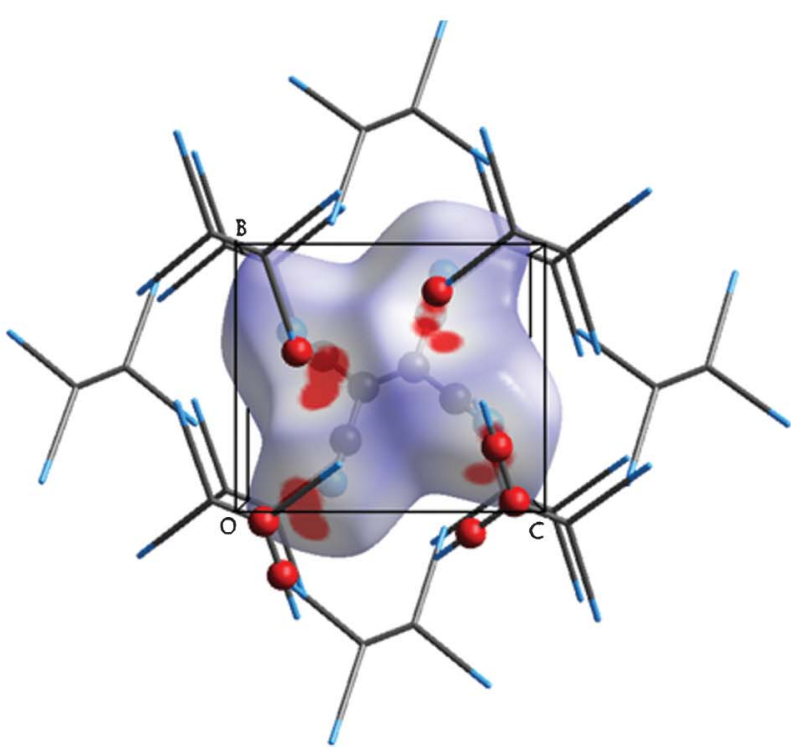

Fig. 1 Cubic and monoclinic polymorphs of TCNE. The central molecules are surrounded by 12 nearest neighbors. The cubic structure is viewed down the $\boldsymbol{b}$ unit cell axis and the monoclinic structure is viewed down the $\boldsymbol{a}$ unit cell axis. Also shown are the Hirshfeld $\mathrm{D}_{\text {norm }}$ surfaces (see Section $\mathbf{3 . 3}$ for explanation). Red atoms correspond to shorter than van der Waals distance close contacts. $\mathrm{D}_{\text {norm }}$ surfaces were standardized to a color scale with minimum $=-0.038$ and maximum $=2$.

to $4 \mathrm{~K}$ ) cubic to monoclinic evolution at temperatures above $318 \mathrm{~K} .{ }^{17-19,23}$ The pressure-induced phase transitions have also been extensively investigated, ${ }^{6-8,21,22,24-26}$ where the monoclinic phase converts to an intermediate 'disordered' metastable phase when pressurized between 2.0-6.3 GPa. Upon release of pressure, the intermediate phase is followed by conversion to either a) the cubic phase (provided sufficient time under high pressure) or b) the original monoclinic phase.

Finally, high pressure polymerization reactions of TCNE at room temperature have been observed when starting from either the monoclinic or cubic phases. ${ }^{6-8,21,22,24-26}$ The most recent investigations indicate that when using no pressure medium, the monoclinic phase completely polymerizes above $6.4 \mathrm{GPa}$ while the cubic phase is stable up to $14 \mathrm{GPa}^{22}$ Interestingly, the polymerized material is a form of amorphous carbon with $52 \%$ less nitrogen than crystalline $\mathrm{TCNE}$, and the $\mathrm{C}: \mathrm{N}$ ratio obtained from neutron/X-ray diffraction was $7: 1$ for the polymerized product compared to $3: 2$ for the original material. $^{7}$

Attempts have been made to explain the temperature and pressure dependence of the monoclinic and cubic phases using quasi-harmonic dynamical calculations with simple empirical force fields. It was determined from those calculations that the monoclinic phase was the more stable of the two phases at elevated temperatures. The reasoning was that the monoclinic polymorph has a higher degree of vibrational entropy compared to the cubic phase, resulting in a lower free energy. ${ }^{27}$ The phase diagram developed from this investigation also demonstrated that the cubic phase is never energetically favorable at ambient pressure. This may be unlikely due to the fact that the cubic phase is obtained via controlled growth at low temperature. Though many qualitative trends of TCNE were replicated, several problems exist with the methodology of these calculations, as pointed out by the author ${ }^{27}$ and others. ${ }^{28}$ Further attempts were made to improve upon these calculations by including the electrostatic interactions within the crystal, but only the monoclinic phase was investigated, so a reliable comparison between the two polymorphs was never performed. ${ }^{28}$

In order to elucidate the energetic variations leading to the temperature dependent phase transition of TCNE, the DFT $+v d W$ method is used in this study to explore the free energies of the cubic and monoclinic polymorphs as a function of temperature. We find that the DFT+vdW approach leads to excellent agreement of the unit cell volumes compared to experiment. The temperature-induced phase transition between cubic and monoclinic polymorphs of TCNE is attributed to the difference in the intermolecular close contacts between these two polymorphs. Finally, a temperature dependent HR-FTIR investigation of the vibrational lifetimes of the $\mathrm{C} \equiv \mathrm{N}$ vibrons confirms the theoretical findings.

\section{Methodology}

\subsection{Computational details}

The density functional theory ${ }^{29}$ method implemented in the program CASTEP ${ }^{30}$ was used in the investigation. Normconserving potentials were employed for $\mathrm{C}$ and $\mathrm{N}$, where valence electrons included $2 \mathrm{~s}^{2} 2 \mathrm{p}^{2}$ and $2 \mathrm{~s}^{2} 2 \mathrm{p}^{3}$, respectively. The plane wave basis set cutoff was $770 \mathrm{eV}$. The use of a relatively high plane wave basis set cutoff is to ensure that the total energy and unit cell volume converge, as demonstrated in a similar study of crystalline indole. ${ }^{31}$ The $k$-point grid was kept to maintain a spacing of $c a$. $0.07 \AA^{-1}$. The GGA functional of Perdew, Burke, and Ernzerhof $(\mathrm{PBE})^{32}$ was used. The convergence criteria for total energy, maximum force, maximum stress, maximum 
displacement, and SCF iterations were $5 \times 10^{-6} \mathrm{eV}$ atom ${ }^{-1}$, $0.01 \mathrm{eV} \AA^{-1}, 0.02 \mathrm{GPa}, 5 \times 10^{-4} \AA$ and $5 \times 10^{-7} \mathrm{eV}$ atom $^{-1}$, respectively. The phonon density of states was calculated with CASTEP's implementation of density functional perturbation theory (DFPT). ${ }^{33}$ A $k$-spacing of $0.0286 \AA^{-1}$ was employed in sampling the vibrational density of states with sufficient resolution.

The DFT+vdW method ${ }^{34}$ was used to account for the ubiquitous van der Waals (vdW) interactions, known to be important for molecular crystals. ${ }^{35-37}$ In the DFT+vdW approach, both the vdW $C_{6}$ coefficients and the vdW radii are determined dynamically, based on the Hirshfeld partitioning of the self-consistent electron density of a given structure. This is of particular importance for molecular crystals, where non-conventional bonding patterns are often present.

The Hirshfeld surfaces and fingerprint plots were produced in the program Crystal Explorer, ${ }^{38}$ where the vdW radii are derived from Bondi. ${ }^{39}$ Initial structures for each calculation were taken from the corresponding X-ray investigations of each polymorph. ${ }^{12,20}$

We note that recent studies ${ }^{40,41}$ suggest that a many-body approach may be necessary for an accurate determination of the intermolecular binding energies. The present pairwise method for $\mathrm{vdW}$ interactions has been shown to reliably model the structures and energetics of other molecular crystals (indole, naphthalene, anthracene, tetracene, and pentacene) within $\sim 0.1 \mathrm{eV}$ variation from experiment. ${ }^{31,42}$

\subsection{HR-FTIR}

TCNE was purchased from Sigma-Aldrich (T8809-98\%). The monoclinic phase was obtained via sublimation according to ref. 18 and 19. The cubic phase was obtained from ethyl acetate solution as described elsewhere. ${ }^{12-15}$ Spectra were obtained using a Bruker HR-120 FTIR at a resolution of $0.1 \mathrm{~cm}^{-1}$. The polycrystalline samples were mounted onto a closed cycle $\mathrm{He}$ cryostat and coupled into the sample compartment of the FTIR with cassegrain optics as previously described. ${ }^{43}$ Only intense isolated bands with high Lorentzian character $(>85 \%)$ were analyzed. Bruker's OPUS software was used to fit a sum of Lorentzian bands ${ }^{44}$ to the IR lineshapes as described elsewhere. $^{45,46}$

\section{Results and discussion}

\subsection{Structure}

The ability of the PBE+vdW method to accurately model the two crystalline phases of TCNE is demonstrated in Table 1 and
Fig. 2. Table 1 demonstrates that only $1 \%$ and $3 \%$ variations with experiment exist for the unit cell parameters, density and molar volumes of the monoclinic and cubic phases respectively. $\mathrm{X}$-ray studies ${ }^{12,20}$ show that the monoclinic phase has a molar volume that is $\sim 5 \%$ larger than that of the cubic phase. The calculated $(\mathrm{PBE}+\mathrm{vdW})$ molar volume of the monoclinic phase is $\sim 4 \%$ greater than that of the cubic phase, improving upon the previous simulation study ${ }^{27}$ which gave just a $1 \%$ difference.

Fig. 2 examines the details of the computed molecular structures as compared to X-ray diffraction (XRD) and neutron structure determinations. In both the monoclinic and cubic phases, the computed values for the $\mathrm{C} \equiv \mathrm{N}$ distances are $\sim 1.16 \AA$, whereas the corresponding values from XRD are $\sim 1.13 \AA$ for both phases, resulting in a $2.6 \%$ difference. On the other hand, available neutron diffraction data at $295 \mathrm{~K}$ and $5 \mathrm{~K}$ for the monoclinic phase gave $\mathrm{C} \equiv \mathrm{N}$ distances of $1.156 \AA^{47}$ and $1.150 \AA^{18}$ respectively, agreeing well with the DFT results (to within 1\%). The observed shortening of the $\mathrm{C} \equiv \mathrm{N}$ bonds in the XRD structures of TCNE and other structures has previously been addressed using high-angle data ${ }^{48}$ and double-atom refinement. ${ }^{12}$ It is important to note that the bond lengths obtained from the calculations and neutron data correspond to the distances between atomic nuclei, whereas those from XRD experiments correspond to the centers of the electron densities for each atom. These two sets of data may not coincide in cases where polar or high order bonds are involved. ${ }^{12,49}$ One way to correct for this in XRD experiments is with a double atom approach, where the electron densities for the core and shell electrons are refined separately. When applied to the cubic polymorph, the structure obtained from the double atom refinement corresponds well with DFT, yielding a "corrected" $\mathrm{C} \equiv \mathrm{N}$ length of $1.166 \AA$. $^{12}$

The same case can be made for the $\mathrm{C}=\mathrm{C}$ and $\mathrm{C}-\mathrm{C}$ bond variation between $\mathrm{X}$-ray and $\mathrm{DFT}$. The $\mathrm{C}=\mathrm{C}$ bond's electron density is contracted towards the center of the bond, resulting in a "shorter" bond length when determined via XRD $\left(1.32 \AA^{20}\right)$. With that being said, both neutron diffraction ${ }^{18,47}$ and DFT show the $\mathrm{C}=\mathrm{C}$ bond to be $1.38 \AA$, and the double atom refinement for the cubic phase ${ }^{12}$ shows it to be $1.36 \AA$. The $\mathrm{C}-\mathrm{C}$ bond is "elongated" when observed via XRD, due to the withdrawal of electron density into the neighboring high order/ polar bonds. In contrast, the $\mathrm{C}-\mathrm{C}$ bond lengths obtained from neutron, DFT, and double atom refinement are all consistently shorter (see Fig. 2). The excellent agreement achieved between the calculations, neutron diffraction, and double atom refinement molecular geometries, as well as the XRD and calculated

Table 1 Crystallographic and energetic parameters of the cubic and monoclinic phases of TCNE. All energetic terms are reported per molecular unit $\left(\mathrm{C}_{6} \mathrm{~N}_{4}\right) . \Delta H_{\text {lattice }}\left(-\Delta H_{\text {sub }}\right)$ is obtained from the difference of the total energy of the crystal and the isolated molecule, including the respective zero-point energies

\begin{tabular}{lccrc}
\hline & PBE+vdW monoclinic & XRD monoclinic $^{20}$ & PBE+vdW cubic \\
\hline$a[\AA]$ & 7.69 & 7.51 & 9.83 & 9.83 \\
$b[\AA]$ & 6.17 & 6.21 & 9.83 & 9.74 \\
$c[\AA]$ & 6.96 & 7.00 & 9.74 \\
Molar volume $\left[\AA^{3}\right]$ & 164.9 & 163.2 & 158.52 & 154.00 \\
Density $\left(\mathrm{g} \mathrm{cm}^{-3}\right)$ & 1.300 & 1.313 & 1.342 & 1.383 \\
Zero point energy $(\mathrm{eV})$ & 1.23 & & -0.97 \\
$\Delta H_{\text {lattice }}(\mathrm{eV})$ & -0.93 & & & \\
\hline
\end{tabular}



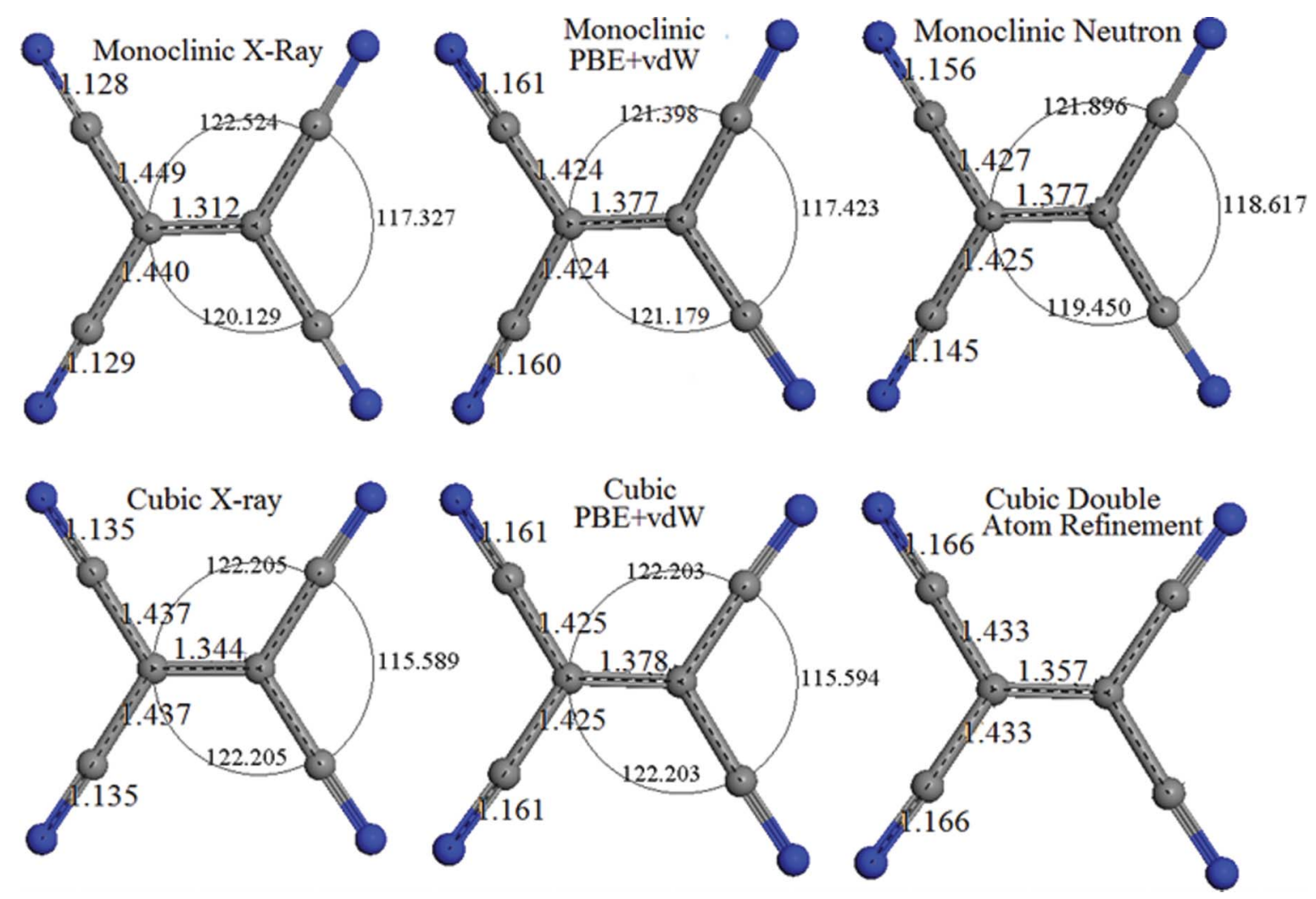

Fig. 2 TCNE molecular geometry in monoclinic and cubic crystalline phases as derived from X-ray spectroscopy, neutron diffraction, and the $\mathrm{PBE}+\mathrm{vdW}$ method. The measurements come from the following references: monoclinic X-ray at $295 \mathrm{~K},{ }^{20}$ monoclinic neutron at $5 \mathrm{~K},{ }^{18}$ cubic $\mathrm{X}$-ray and double atom refinement at $295 \mathrm{~K} .{ }^{12}$

unit cell parameters, displays the usefulness of the present $\mathrm{PBE}+\mathrm{vdW}$ methodology.

\subsection{Energetics}

As mentioned, previous rigid body calculations of TCNE with a simple empirical force field attempted to demonstrate the relative stabilities of the two crystalline phases with respect to pressure and temperature, resulting in a calculated phase diagram. ${ }^{27}$ It was concluded that the static potentials of both the monoclinic and cubic phases were very close across the entire temperature and pressure range, but that the monoclinic phase exhibited increased energetic stability at elevated temperatures; this was attributed to increased vibrational entropy when compared to its cubic cousin. While the qualitative phase stabilities trends were followed at elevated pressures, the monoclinic phase was calculated always to be energetically favorable at pressures below $\sim 0.5 \mathrm{GPa}$ regardless of temperature. This would mean that the cubic phase is always metastable at ambient pressure.

While the present study confirms that the stability of the two phases are similar, it is shown in Table 1 and Fig. 3a that the cubic phase is energetically more favorable at $0 \mathrm{~K}$ than the monoclinic phase by $0.04 \mathrm{eV}$ (contrary to the previous study ${ }^{27}$ ). This difference in crystal packing energies is in agreement with the experimental observation that slow crystallization of TCNE from solution at low temperatures results in the cubic phase. Yet, the difference in energy of the two phases is so small that fast crystallization at temperatures suitable for cubic phase growth can result in the monoclinic polymorph. ${ }^{17}$

We used harmonic phonon calculations ${ }^{33}$ of the two polymorphs to determine the phonon density of states (DOS), providing insight into the temperature dependence of the enthalpy, entropy, and therefore free energy (see Fig. 3). The results of these calculations show that the cubic phase is energetically favorable at temperatures below $160 \mathrm{~K}$, and that the monoclinic phase becomes the preferred polymorph at higher temperatures (see Fig. 3a). It should be pointed out that, while the cubic to monoclinic transition temperature is found at $160 \mathrm{~K}$ in Fig. 3a, no temperature induced transition is observed experimentally until $318 \mathrm{~K}$. This fact can be attributed to the large phase transition barrier $\left(230 \pm 20 \mathrm{~kJ} \mathrm{~mol}^{-1}[\sim 2.38 \mathrm{eV}]\right)$ which hinders molecular reorientation at low temperatures, ${ }^{17}$ causing metastability of the cubic phase between 160 and $318 \mathrm{~K}$. The large transition barrier also explains the fact that the cubic phase retains its symmetry at room temperature, even after several months. ${ }^{23}$ On the other hand, the transition barrier, in addition to the very small difference in energetics, explains why the inverse phase transition (from monoclinic to cubic) is not observed when the sample temperature drops below $318 \mathrm{~K}$.

The temperature dependence of the entropy and enthalpy are presented in Fig. $3 \mathrm{~b}$ and $3 \mathrm{c}$ respectively. Fig. 3b demonstrates that the entropy temperature dependence of the monoclinic phase is always greater than that of the cubic phase. On the other hand, Fig. $3 \mathrm{c}$ shows that the temperature dependence of the enthalpy of the monoclinic phase is greater than that of the cubic 


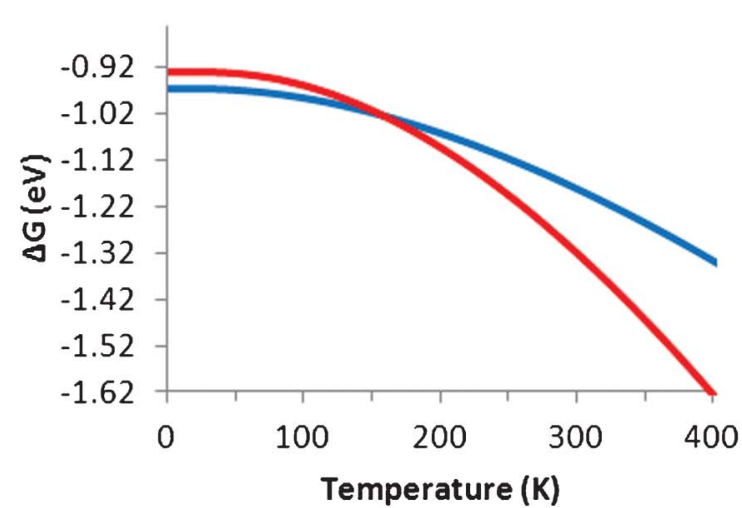

(a)

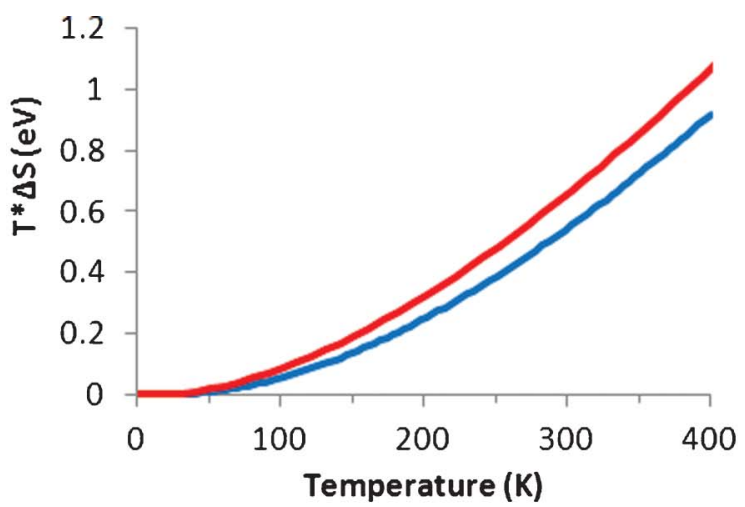

(b)

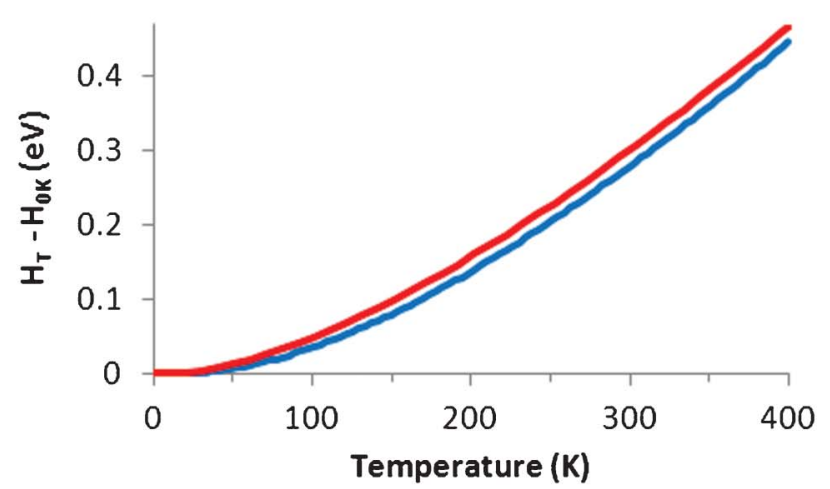

(c)

Fig. 3 Thermodynamic properties of the monoclinic and cubic phases as a function of temperature, calculated with PBE+vdW. Blue represents the cubic phase and red represents the monoclinic phase. All quantities are reported per molecular unit. (a) Free energy as a function of temperature. (b) Entropy change as a function of temperature. (c) Enthalpy change as a function of temperature relative to $0 \mathrm{~K}$.

phase below $150 \mathrm{~K}$, but becomes equivalent at higher temperatures. Both of these trends contribute to the lower free energy of the monoclinic phase at elevated temperatures.

The phonon DOS of the two polymorphs below $10 \mathrm{THz}$ is compared in Fig. 4. It is known that low-frequency phonons, particularly those below $6 \mathrm{THz}$, assert heavier weight in changing the vibrational entropy component of the free energy than those of higher energy. ${ }^{27}$ Therefore, any shift in the density of states below $6 \mathrm{THz}$ will have a larger impact on the free energy than shifts above $6 \mathrm{THz}$. As evident in Fig. 4, the monoclinic phase has a higher phonon DOS in the low frequency regions, particularly below $2 \mathrm{THz}$, compared to the cubic phase. The substantially greater density of vibrational states below $2 \mathrm{THz}$ in the monoclinic phase favors this phase's stability at elevated temperatures due to increased vibrational entropy.

\subsection{Increased intermolecular coupling leads to higher entropy for the monoclinic polymorph}

While the energies of the two polymorphs are very close, their individual crystal structures are quite different, as shown in
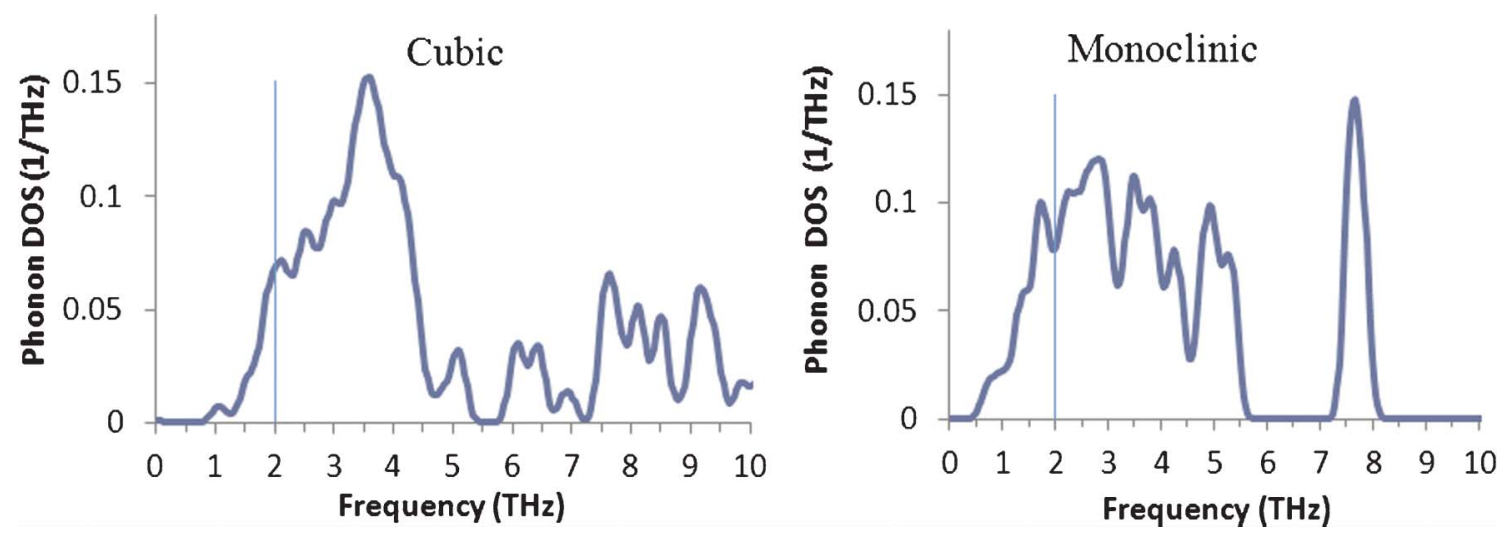

Fig. 4 Phonon density of states for the cubic and monoclinic phases of TCNE, computed with PBE+vdW. 
Fig. 1. In order to further rationalize the increased entropy of the monoclinic structure with respect to the cubic polymorph, we calculated the Hirshfeld surfaces of both phases. Hirshfeld surfaces sample the electron density surrounding the molecules and correlate intermolecular close contacts occurring at the surface to specific atom types. This provides the ability to demonstrate specific atom-atom intermolecular interactions, as well as providing a quantitative value to the amount of a given contact type with respect to all others in a $2 \mathrm{D}$ fingerprint plot. $^{50-52}$

The $\mathrm{D}_{\text {norm }}$ Hirshfeld surfaces generated from the crystalline X-ray structures of both polymorphs surrounded by the 12 nearest neighbors are shown in Fig. 1. There are essentially only $\mathrm{C} \cdots \mathrm{N}$ and $\mathrm{N} \cdots \mathrm{N}$ contacts existing within the two polymorphs (see Figures a and $\mathrm{b}$ in supplementary data $\uparrow$ for the decomposed $\mathrm{D}_{\text {norm }}$ surfaces, fingerprint plots, and close contact histograms). Here, color is the index, where the bright red spots on the $\mathrm{D}_{\text {norm }}$ surfaces represent the $\mathrm{C} \cdots \mathrm{N}$ contacts of neighboring molecules that are shorter than the vdW radius of the two interacting atoms, while the white areas on the surfaces represent contacts occurring at vdW separations and the blue represents contacts occurring beyond the vdW radii. ${ }^{39}$

Bright blue tips visible at either end of the cubic structure's $\mathrm{D}_{\text {norm }}$ surface in Fig. 1 are indicative of voids present in the structure and are not observed for the monoclinic phase (also see $\mathrm{D}_{\mathrm{e}}$ surface in Figure a of supplementary data). When the temperature is lowered or pressure is increased, these voids aid in denser packing. ${ }^{21,22}$ The $\mathrm{D}_{\text {norm }}$ surfaces in Fig. 1 also demonstrate that the monoclinic phase possess $20 \mathrm{C} \cdots \mathrm{N}$ contacts which occur at distances shorter than vdW separations (bright red circles). Conversely, the cubic polymorph contains only 16 centers of $\mathrm{C} \cdots \mathrm{N}$ contacts which occur at shorter than vdW separations. As a result of the higher number of shorter than $\mathrm{vdW}$ distance $\mathrm{C} \cdots \mathrm{N}$ contacts in the monoclinic phase, and its lack of crystalline voids, it would be reasonable to expect increased intermolecular coupling between the $\mathrm{C} \equiv \mathrm{N}$ vibrational modes under ambient conditions when compared to the cubic phase.

In order to verify this hypothesis, we carried out temperaturedependent measurements of the IR linewidths for both the cubic and monoclinic polymorphs. In general, it is known that variations in the close contact interactions (both length and number) can affect the strength of intermolecular mode couplings and lead to shifts in the phonon density of states, each of which can affect the vibrational dynamics that determine the vibrational linewidth. It has been demonstrated via high pressure IR experiments on TCNE that since the cyano groups are oriented outward from the central $\mathrm{C}=\mathrm{C}$ bond, that the $\mathrm{C} \equiv \mathrm{N}$ stretching frequencies are particularly sensitive to molecular approach and rearrangement. ${ }^{21}$ In addition to the preceding statements, since all shorter than vdW interactions were determined to be between nitrogen and carbon atoms, particular attention was paid to the characteristic temperature dependencies of the $\mathrm{C} \equiv \mathrm{N}$ vibrational linewidths.

Since the monoclinic phase has a greater number of shorter than van der Waals $\mathrm{C} \cdots \mathrm{N}$ contacts under ambient conditions, faster depopulation/dephasing of the $\mathrm{C} \equiv \mathrm{N}$ modes should occur under such conditions, i.e., conceivably more ways exist to strongly couple modes involving nitrogen to neighboring molecules in the monoclinic phase under ambient conditions, so the full width at half max (FWHM) of these bands should be broader above the free energy transition temperature. On the other hand, as the temperature is lowered and the cubic phase packs more efficiently, the intermolecular mode couplings and bandwidths of the cubic phase should approach those of the monoclinic phase.

The temperature dependent linewidths (FWHM) for the IR active $\mathrm{C} \equiv \mathrm{N}$ stretching vibrons are presented in Fig. 5. The

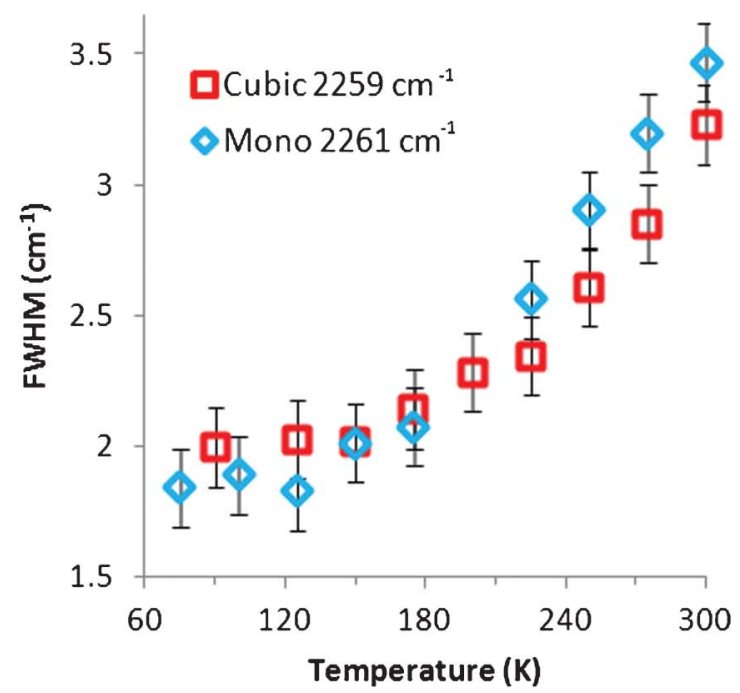

(a)

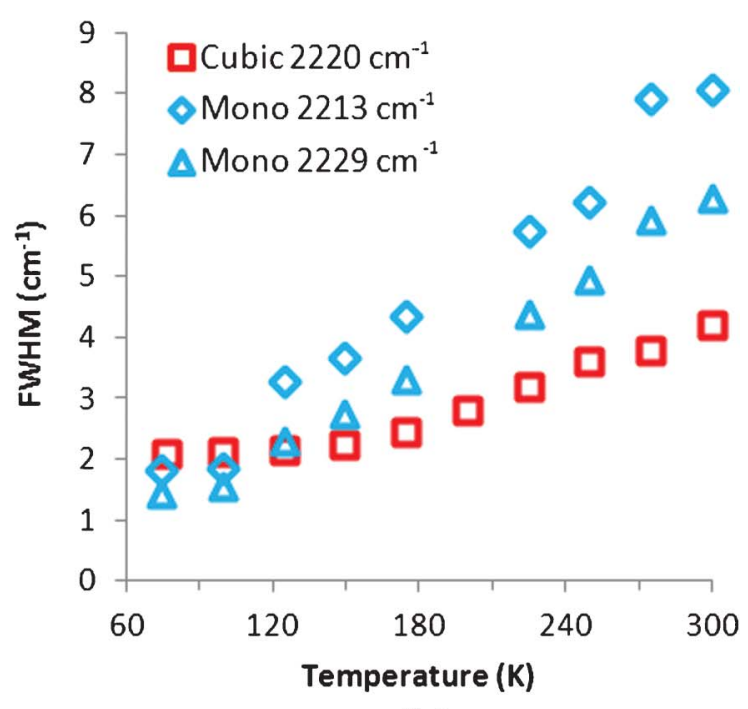

(b)

Fig. 5 Temperature dependent linewidths (FWHM) of the $\mathrm{C} \equiv \mathrm{N}$ stretching modes obtained from HR-FTIR measurements for the cubic and monoclinic polymorphs. Error bars are $\pm 0.15 \mathrm{~cm}^{-1}$ and exist within the symbols of (b). The differing number of modes between the two phases results from the two $\mathrm{C} \equiv \mathrm{N}$ stretching modes of the free molecule $\left(\mathrm{B}_{1 \mathrm{u}}\right.$ and $\left.\mathrm{B}_{2 \mathrm{u}}\right)$ splitting into four modes $\left(2 \mathrm{~A}_{\mathrm{u}} / 2 \mathrm{~B}_{\mathrm{u}}\right)$ in the monoclinic phase while translating into two $\mathrm{F}_{\mathrm{u}}$ modes in the cubic phase. ${ }^{21}$ The fourth $\mathrm{C} \equiv \mathrm{N}$ stretching band of the monoclinic phase is a shoulder and is not included in the analysis (see figure c in supplementary data†). 
monoclinic and cubic structures show distinctly different linewidth temperature dependencies, with the monoclinic phase possessing broader bands at temperatures $>\sim 160 \mathrm{~K}$ (remarkably similar to the free energy crossing, see Fig. 3a). This difference in linewidth temperature dependence is associated with the higher number of shorter than $\mathrm{vdW} C \cdots \mathrm{N}$ contacts in the monoclinic phase at high temperature compared to the higher packing ability of the cubic phase at low temperature. Therefore, the noted variations in the number and distances of intermolecular close contacts observed in the Hirshfeld surface analysis are shown to have a dramatic effect on the manner/rate of energetic depopulation.

\section{Conclusions}

The density-functional theory with van der Waals interactions $(\mathrm{DFT}+\mathrm{vdW})$ method is capable of yielding excellent agreement with experiment for the two established polymorphs of TCNE. It was found that, for the cubic and monoclinic polymorphs of TCNE, the enthalpies of formation at $0 \mathrm{~K}$ differ by only $0.04 \mathrm{eV}$. The cubic phase is determined to be slightly more stable at $0 \mathrm{~K}$, but becomes less favorable in free energy at $160 \mathrm{~K}$. The Hirshfeld surface analysis of the two crystalline polymorphs demonstrates varying qualitative interactions between the neighboring molecules of the two phases, but reveals only slight quantitative variation between the fractions of intermolecular close contact interactions. A higher number of shorter than vdW distance close contacts was observed within the monoclinic phase as compared to that for the cubic phase, underlining the increased vibrational entropy at elevated temperatures within the monoclinic phase, and serving as the primary driving force for the temperature induced phase transition.

\section{Acknowledgements}

Bohdan Schatschneider thanks the Eberly Science Foundation for their generous support of this research. Jian-jie Liang thanks the Accelrys management for allocating resources in supporting the related researches. Alexandre Tkatchenko was supported by the European Research Council (ERC Starting Grant VDWCMAT).

\section{References}

1 J. P. M. Lommerse, W. D. S. Motherwell, H. L. Ammon, J. D. Dunitz, A. Gavezzotti, D. W. M. Hofmann, F. J. J. Leusen, W. T. M. Mooij, S. L. Price, B. Schweizer, M. U. Schmidt, B. P. Van Eijck, P. Verwer and D. E. Williams, Acta Crystallogr., Sect. B: Struct. Sci, $2000, \mathbf{5 6}, 697$.

2 W. D. S. Motherwell, H. L. Ammon, J. D. Dunitz, A. Dzyabchenko, P. Erk, A. Gavezzotti, D. W. M. Hofmann, F. J. J. Leusen, J. P. M. Lommerse, W. T. M. Mooij, S. L. Price, H. Scheraga, B. Schweizer, M. U. Schmidt, B. P. van Eijck, P. Verwer and D. E. Williams, Acta Crystallogr., Sect. B: Struct. Sci., 2002, 58, 647.

3 G. M. Day, W. D. S. Motherwell, H. L. Ammon, S. X. M. Boerrigter, R. G. Valle Della, E. Venuti, A. Dzyabchenko, J. D. Dunitz, B. Schweizer, B P. van Eijck, P. Erk, J. C. Facelli, V. E. Bazterra, M. B. Ferraro, D. W. M. Hofmann, F. J. J. Leusen, C. Liang, C. C. Pantelides, P. G. Karamertzanis, S. L. Price, T. C. Lewis, H. Nowell, A. Torrisi, H. A. Scheraga, Y. A. Arnautova, M. U. Schmidt and P. Verwer, Acta Crystallogr., Sect. B: Struct. Sci., 2005, 61, 511.

4 G. M. Day, T. G. Cooper, A. J. Cruz-Cabeza, K. E. Hejczyk, H. L. Ammon, S. X. M. Boerrigter, J. S. Tan, R. G. Della Valle, E. Venuti, J. Jose, S. R. Gadre, G. R. Desiraju, T. S. Thakur, B. P. van Eijck,
J. C. Facelli, V. E. Bazterra, M. B. Ferraro, D. W. M. Hofmann, M. A. Neumann, F. J. J. Leusen, J. Kendrick, S. L. Price, A. J. Misquitta, P. G. Karamertzanis, G. W. A. Welch, H. A. Scheraga, Y. A. Arnautova, M. U. Schmidt, J. van de Streek, A. K. Wolf and B. Schweizer, Acta Crystallogr., Sect. B: Struct. Sci., 2009, 65, 107.

5 L. A. Chetkina and V. K. Bel'ski, Crystallogr. Rep., 2002, 47, 581.

6 R. Rao, T. Sakuntala, S. K. Deb and R. Mukhopadhyay, J. Phys.: Condens. Matter, 2005, 17, 2633.

7 R. Mukhopadhyay and S. L. Chaplot, J. Phys.: Condens. Matter, 2002, 14, 759 .

8 H. Yamawaki, M. Sakashita and K. Aoki, Phys. Rev. B: Condens. Matter, 1996, 53, 11403.

9 M. Batley and L. A. Lyons, Mol. Cryst., 1968, 3, 357.

10 D. Jérôme, A. Mazaud, M. Ribault and K. Bechgaard, J. Phys., Lett., 1980, 41(4), 95-98.

11 D. A. Pejaković, C. Kitamura, J. S. Miller and A. J. Epstein, J. Appl. Phys., 2002, 91, 7176.

12 R. G. Little, D. Paulter and P. Coppens, Acta Crystallogr., Sect. B: Struct. Crystallogr. Cryst. Chem., 1971, 27, 1493.

13 P. J. Becker and P. Coppens, J. Am. Chem. Soc., 1973, 95, 7604.

14 J. Murgich and S. Pissanetzky, J. Chem. Phys., 1975, 62, 92.

15 R. Mukhopadhyay, S. L. Chaplot and K. R. Rao, Phys. Status Solidi A, 1985, 92, 467.

16 S. Onda, R. Ikeda, D. Nakumura and M. Kubo, Bull. Chem. Soc. Jpn., 1969, 42, 2740.

17 R. Mukhopadhyay, S. L. Chaplot and K. R. Rao, Phys. Status Solidi A, 1985, 92, 467.

18 S. L. Chaplot, A. Mierzejewski and G. S. Pawley, Acta Crystallogr., Sect. C: Cryst. Struct. Commun., 1984, 40, 663.

19 S. L. Chaplot, A. Mierzejewski, G. S. Pawley, J. Lefebvre and T. Luty, J. Phys. C: Solid State Phys., 1983, 16, 625.

20 D. A. Bakoe and K. N. Trueblood, Z. Kristallogr., 1960, 113, 1.

21 H. Yamawaki, K. Aoki, Y. Kakudate, M. Yoshida, S. Usuba and S. Fujiwara, Chem. Phys. Lett., 1992, 198, 183.

22 R. Mukhopadhyay, S. K. Deb, A. Das and S. L. Chaplot, Solid State Commun., 2009, 149, 1914.

23 A. Mierzejewski and S. L. Chaplot, Conf. Int. Raman Spectrosc., 7th, 1980, pp. 46-7.

24 S. L. Chaplot, A. Mierzejewski and G. S. Pawley, Mol. Phys., 1985, 56, 115.

25 S. L. Chaplot and R. Mukhopadhyay, Phys. Rev. B, 1986, 33, 5099.

26 P. Sahu, K. G. Rajan, M. Yousuf, R. Mukhopadhyay, S. L. Chaplot and R. Rao, Pramana, 1989, 33, 667.

27 S. L. Chaplot, Phys. Rev. B, 1987, 36, 8471.

28 T. H. M. van den Berg and A. van der Avoird, J. Phys.: Condens. Matter, 1989, 1, 4047.

29 P. Hohenberg and W. Kohn, Phys. Rev., 1964, 136, B864.

30 S. J. Clark, M. D. Segall, C. J. Pickard, P. J. Hasnip, M. J. Probert, K. Refson and M. C. Payne, Z. Kristallogr., 2005, 220, 567.

31 B. Schatschneider and J. J. Liang, J. Chem. Phys., 2011, 135, 164508.

32 J. P. Perdew, K. Burke and M. Ernzerhof, Phys. Rev. Lett., 1996, 77, 3865.

33 S. Baroni, S. de Gironcoli, A. dal Corso and P. Giannozzi, Rev. Mod. Phys., 2001, 73, 515.

34 A. Tkatchenko and M. Scheffler, Phys. Rev. Lett., 2009, 102, 073005.

35 M. A. Neumann and M. A. Perrin;, J. Phys. Chem. B, 2005, 109, 9810.

36 H. C. Chan, J. Stephen; Kendrick and F. J. J. Leusen, Phys. Chem. Chem. Phys., 2011, 13, 20361.

37 N. Marom, A. Tkatchenko, S. Kapishnikov, L. Kronik and L. Leiserowitz; Cryst. Growth Des., 2011, 11, 3332.

38 S. K. Wolff, D. J. Grimwood, J. J. McKinnon, M. J. Turner, D. Jayatilaka and M. A. Spackman, CrystalExplorer (Version 2.2), University of Western Australia, 2010.

39 J. J. McKinnon, D. Jayatilaka and M. A. Spackman, Chem. Commun., 2007, 3814.

40 R. Podeszwa, B. M. Rice and K. Szalewicz, Phys. Rev. Lett., 2008, 101, 115503.

41 O. A. von Lilienfeld and A. Tkatchenko, J. Chem. Phys., 2010, 132, 234109.

42 B. Schatschneider, J. J. Liang and A. Tkatchenko, "Simulated highpressure dynamics of polyacenes: DFT $+v d W$ study", 2012, to be published, contact for data.

43 R. Bini, R. Ballerini, G. Pratesi and H. J. Jodl, Rev. Sci. Instrum., 1997, 68, 3154. 
44 Y. Liu, J. Lin, G. Huang, Y. Guo and C. Duan, J. Opt. Soc. Am. B, 2001, 18, 666.

45 B. Schatschneider, Structural and Vibrational Dynamics of Molecular Solids Under Variable Temperature and Pressure, PhD. Thesis, 2008 University of California, Riverside.

46 B. Schatschneider and E. L. Chronister, Chem. Phys. Lett., 2012, 533, 30.

47 S. L. Chaplot, R. Chakravarthy, W. I. F. David and J. Tomkinson, J. Phys.: Condens. Matter, 1991, 3, 9271.

48 P. Coppens, Abstracts A.C.A. Meeting, Ottawa, 1970, p. 65.
49 P. Coppens, T. M. Sabine, R. G. Delaplane and J. A. Ibers, Acta Crystallogr., Sect. B: Struct. Crystallogr. Cryst. Chem., 1969, 25, 2451.

50 M. A. Spackman and D. Jayatilaka, CrystEngComm, 2009, 11, 19.

51 Crystal Explorer Help; http://www.hirshfeldsurface.net/wiki/index.php/ Manual.

52 J. J. McKinnon, M. A. Spackman and A. S. Mitchell, Acta Crystallogr., Sect. B: Struct. Sci., 2004, 60, 627.

53 B. Schatschneider, J. Phelps and S. Jezowski, CrystEngComm, 2011, 13, 7216 . 